\title{
Integrated Architecture for Next-Generation m-Health Services (Education, Monitoring and Prevention) in Teenagers
}

\author{
Marco Mazzola ${ }^{1}$, Pelin Arslan ${ }^{1}$, Gabriela Cândea ${ }^{2}$, Ciprian Radu², \\ Massimiliano Azzolini ${ }^{3}$, Cristiana Degano ${ }^{3}$, and Giuseppe Andreoni ${ }^{1}$ \\ ${ }^{1}$ Politecnico di Milano, Design Department, Milan, Italy \\ \{marco.mazzola, giuseppe.andreoni\}@polimi.it, \\ pelinarslan1@gmail.com \\ ${ }^{2}$ ROPARDO S.R.L., Research\&Development, Sibiu, Romania \\ \{gabriela.candea, ciprian.radu\} @ropardo.ro \\ ${ }^{3}$ Gruppo SIGLA S.r.l., Research\&Development, Genova, Italy \\ \{massimiliano.azzolini, cristiana.degano\}@grupposigla.it
}

\begin{abstract}
Obesity and other lifestyle-related illness are among the top healthcare challenges in Europe. The rapid development of the ICT, and in particular mobile technologies offers an important opportunity for introducing the possibility of a new technological framework. In this paper, the PEGASO system is presented. It will be based on a mobile, social and networked gaming platform, considered as a powerful tool to actively engage the younger population in activities that will stimulate healthier choices in their daily lives. The PEGASO project will implement the User Centred Design approach by considering our target population (i.e. teenagers) at the centre of the system in a palingenetic process. Smartphone is the first and key sensor system. The mobile device also acts as communication gateway towards the other sensors. Basic services, such as those related to location and basic motion sensors to detect physical activity, are provided through sensors embedded within the smartphone.
\end{abstract}

Keywords: Teenagers Obesity Prevention, Mobile Based Platform, System Architecture.

\section{Introduction}

Obesity and other lifestyle-related illness are among the top healthcare challenges in Europe. Obesity alone accounts for up to $8 \%$ of healthcare costs in the EU[1], as well as wider economy costs associated with lower productivity, lost output and premature death. Childhood overweight and obesity are demonstrated to be strongly related with adult obesity and cardiovascular disease risks, hypertension, metabolic syndrome, fatty liver disease, sleep disturbance and childhood onset type-II diabetes mellitus. [2,3,4]

Obesity in younger age is an alarming predictor for obesity in adulthood, but also entails short term health complications in juvenile age along with greater risk of social 
and psychological problems [5]. Knowing how to stay healthy is not enough to motivate individuals to adopt healthy lifestyles, but relevant progress can be achieved through the use of incentives delivered through a combination of processes and mobile technologies [6]. In this landscape, prevention assumes an absolute relevance, and there is an urgent need for further research in how physical activity, training and nutrition can be protective of obesity. With growing rates of childhood overweight and obesity, it becomes increasingly more important to promote healthy eating as poor dietary behaviors are a known risk factor for the development of obesity [7,8].

The main relevant factors tackling childhood obesity prevention result to be related with the Physical Activity, Nutrition and in behavioural education. Addressing the obesity issues requires a comprehensive approach taking into account the individual's physical-physiological characteristics, personality as well as the social and psychological environments influencing decisions and habits in their everyday life. Great relevance should be given to actions developing awareness and enhancing motivation for changing behaviour towards healthy diet (dietary) and physical activity (active lifestyle) [9].

It is demonstrated that Physical Activitiy is protective of obesity. [10-11]. On the contrary, the relation between sedentary time and obesity is not completely defined, but the increased rates of overweight and obesity among children can be attributed to an increase in sedentary pursuits, in relation of the most evidence of higher caloric intake spent in screen time. This fact is relevant because it explains the relation between time spent in, for example, watching television, and the increased amount of food intake as a main cause of overweight.

The rapid development of the ICT, and in particular mobile technologies, together with their diffusion among the EU populations, offers an important opportunity for facing these issues in an innovative manner introducing the possibility of a new technological framework to re-design the healthcare system model. Moreover, establishing healthy behaviors during childhood seems to be easier and more effective than trying to change unhealthy behaviors during adulthood as motivation may be easier to generate while the child is young [12].

The use of technological devices for an active lifestyle monitoring is widely increased in the recent years. As an example, the use of wearable sensors' network has been explored for their application in obesity and activity monitoring [13];

In addition, commercial solutions of monitoring devices are becoming really popular; prove of that is the release of physical activity monitor systems products such as FitBit ultra, sold for 99.95US\$ by Fitbit [14], and able to monitor journal activity and sleep patterns. The device includes a 3D accelerometer and an altimeter that permit to track the physical activity. The feedback is provided as estimations of the number of steps, distance, floor climbed, and energy expenditure. As a confirmation of the general interest in this area of technological development, the most influencing brands in ICT, as an example Apple and Samsung, has recently decided to enter this market with smart-watches and specific projects with health purposes.

The behavioural management should be also sensitive to social factors as relations with peers through social network media and personal opportunities focused on increasing awareness and personal involvement in the issues of healthy lifestyles 
to contrast body mass excess. In this area, the number of projects approaching the education in nutrition and the development of social platform, dedicated apps and serious games is really relevant.

As an example, KickinKitchen.tv [15] from KidsCook Production of Boston, MA, is a web series and online platform based on a musical sitcom for kids ages 8-15 that promotes healthy eating and lifestyles through the creation of a social online environment, including episodes, weekly challenges and special guests contribution.

Another relevant example are the Apps for Healthy Kids challenge [16], promoted by the United States Department of Agriculture, that challenged software developers, game designers, students to develop software tools and apps to encourage children to health nutrition and physical activity. The competition prize was a non cash recognition based on the popularity among visitors and users reached by the developed software.

Video Gaming, concerning serious gaming or exergames, represents another relevant effort in terms of Obesity prevention among children. As an example, Escape From Diab [17] is a serious video game adventure in healthy eating and exercise, released by Archimage in collaboration with experts at the Children's Nutrition Research Center of Houston's Baylor College of Medicine on the project. The video game was designed as an epic adventure, comparable to the experience of commercial quality video and incorporates a broad diversity of behavior change procedures woven in and around engrossing stories.

In this paper, the PEGASO project, an FP7 EU Integrate Project funded by the European Community, and coordinated by Politecnico di Milano is presented through the description of the adopted methodological approach and the System Architecture.

The PEGASO project aims to create a learning platform capable of educating youth through virtual and real-life games and group challenges, in a "social" approach in which they each influence one another. To address these challenges, PEGASO will develop a multi-dimensional and cross-disciplinary ICT system that includes game mechanics to influence behaviours in order to fight and prevent overweight and obesity in the younger population by encouraging them to become co-producers of their wellness and take an active role in improving it by:

- generating self-awareness (acknowledgement of risks associated to unhealthy behaviours),

- enhancing and sustaining motivation to take care of their health with a short/medium/long term perspective,

- changing behaviour towards a healthy lifestyle based on healthy diet and adequate physical activity.

PEGASO will be based on a mobile, social and networked gaming platform, considered as a powerful tool to actively engage the younger population in activities that will stimulate healthier choices in their daily lives.

In consideration of the above, the PEGASO system framework will address prevention, by offering to teenagers - the primary target of PEGASO - three main functionalities: 
1. Individual \& Environmental Monitoring - This dimension consists of the environmental, behavioural and physiological analysis of young users, through a high level-monitoring platform including wearable sensors and mobile phones, as well as multimedia diaries for the acquisition of physical, behavioural and emotional attitude of adolescents.

2. Feedback System - The second functionality is aimed at providing a feedback in terms of "health status" changes, required actions to undertake and so on. This function will also propose personalized healthy modification of the lifestyle (in terms of diet and/or physical activity), thus promoting the active involvement of adolescents in changing their behaviours.

3. Social connectivity and engagement - The third dimension extends to include a social network where the user can share experiences with a community of peers concerning, e.g. physical activity, food consumptions and everyday habits through different gaming strategies.

\section{Methodological Approach}

The PEGASO project will implement the User Centred Design approach (UCD)[18] by considering our target population (i.e. teenagers) at the centre of the system in a palingenetic process. This approach is useful to motivate and engage users, which is an essential requirement for systems' acceptance and efficacy rather than forcing to accommodate technologies, products, or services. It should be underlined that PEGASO, as tool for prevention, is addressed also to healthy people.

The main target users in the PEGASO project are teenagers; however there are also several actors (who are also secondary users - Figure 1) and products/systems (the inner circle in Figure 1) involved. The ecosystem of stakeholders and enablers is composed of three main parts that are integrated in the user centred PEGASO system:

\section{User Centred Approach}

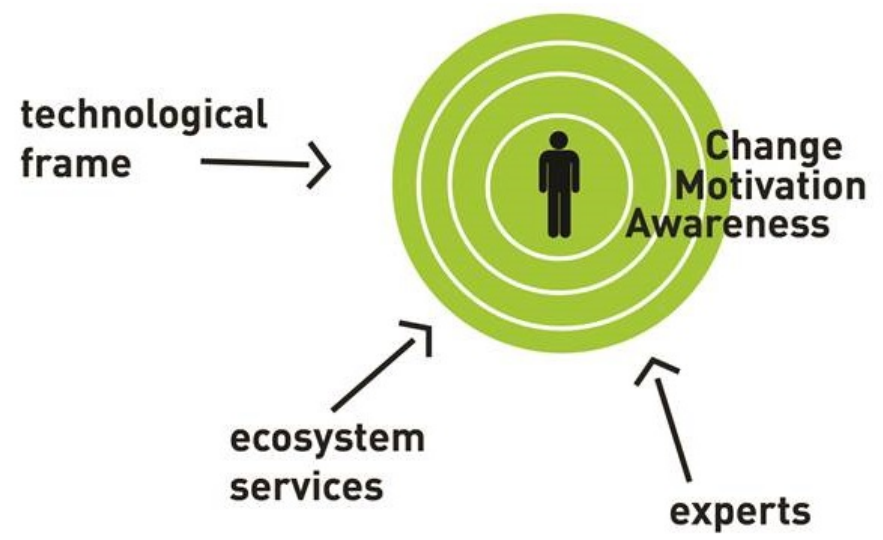

Fig. 1. User Centred Approach adopted in PEGASO 
technological frame (multimedia diaries, embedded sensors systems, mobile \& web platform), services frame (stakeholders services to provide answers to users' needs and desires in real time/not real time, from the health companion to the serious gaming and social experiences) and experts layer (which are knowledgeable groups of people from different disciplines - medical/psychological/educational - able to interact with the system, who provide them with filtered accurate and needed information to reach their PEGASO objective).

Regarding the ecosystem of the PEGASO project, it is important to consider all personal levels of influence of one's prevention in its environment. Figure 2 below is developed on the basis of the Circles of Influence in Self-Management of Chronic Disease [19] and customized to the target needs of PEGASO, i.e. address prevention management in teenagers.

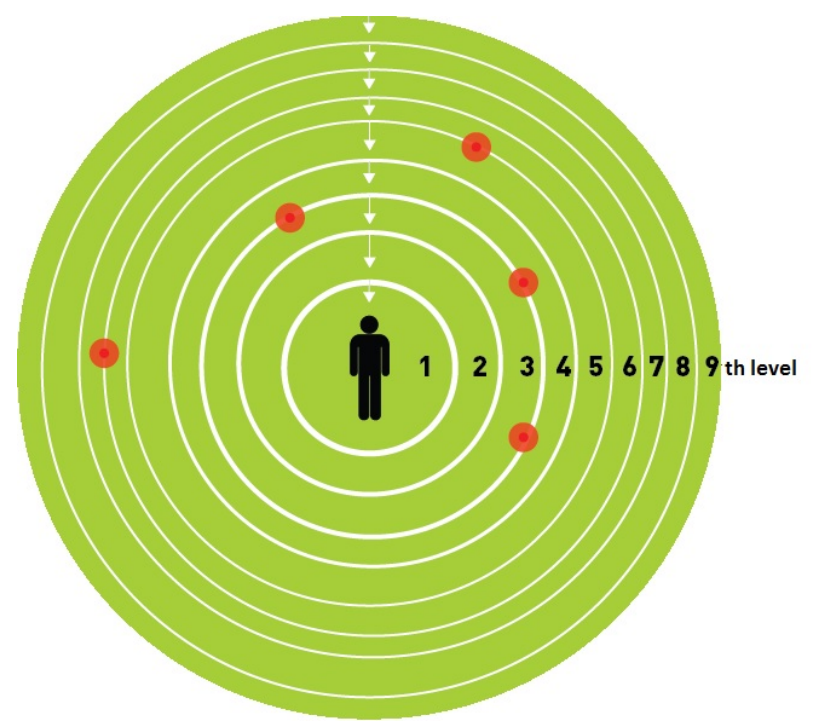

Fig. 2. User's Circles of Influence in Health Management

The circle hierarchy revolving around the end user is not defined in a fixed way; each ring of the concentric circles represents different levels of influence, dispersed around the user: self-management, family involvement, peers influence, experts, school support, community awareness, and environmental measure, industry and policy decisions to the success of the user's prevention management efforts. Therefore for each individual the circles of influence will have different hierarchies, interactions and points (marked in red colour) of decisions for healthy living.

In each circle of influence, the end user has an interaction towards their everyday health. In some circles, the user has the possibility to contribute in decision-making process, in some other they cannot.

Therefore, in the PEGASO project development, it is highly important to focus on the interactions points between end users and levels of their influences, in particular 
where end user is able to give their own decisions, or where the intervention of services and persuasion to healthier lifestyle changes will be acted upon these opportunity areas. This analysis is important to define for user requirements for the design of the PEGASO persuasion strategies.

Moreover, PEGASO considers the various levels towards persuasion for healthcare[20]. Various types of experts and technologies will feed these levels of persuasion towards healthier decisions. There are four different persuasion strategies: awareness of obesity risks, motivation, affective learning and behaviour change.

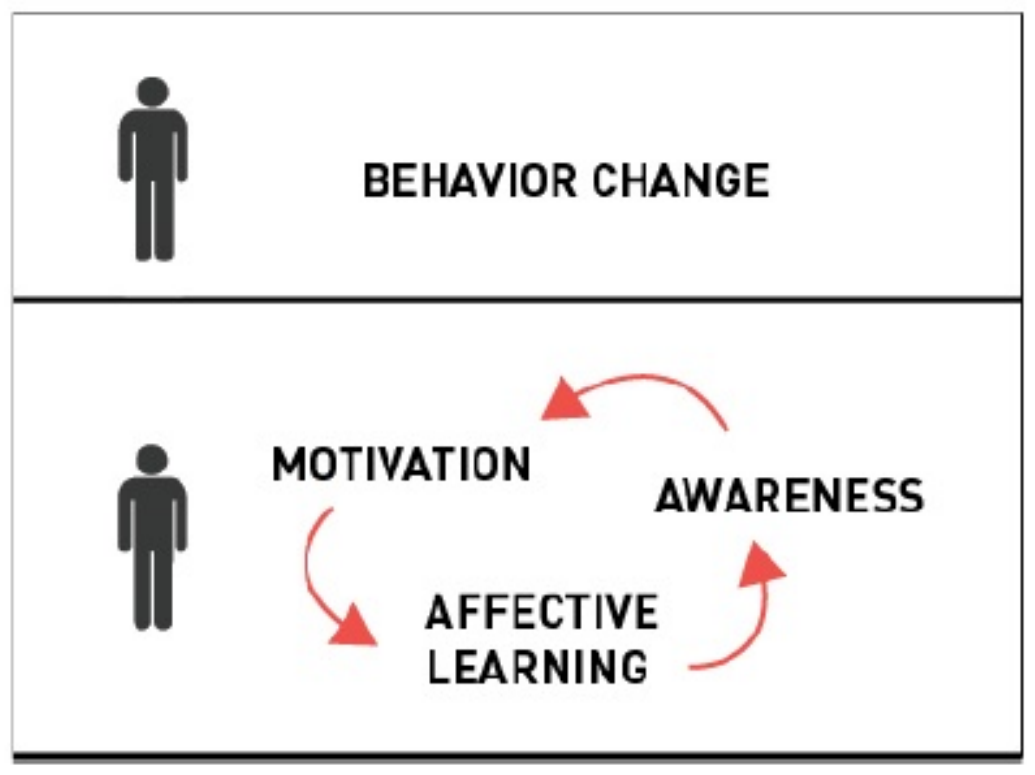

Fig. 3. Persuasion Level for Disease Prevention

- Develop Awareness: Teenagers need to be aware of what they are doing; what is right, and what is wrong for their healthy living. Through developing selfawareness and self-reflection, the user can frame the problem or the opportunity area to act upon or intervene.

- Create Motivation: It is important to motivate teenagers to change their behaviour and keep this activity in a long-term period. This part is quite challenging, since the motivation depends on many factors as well as emotions, psychological environment and personality of teens.

- Enable Behaviour Change: Once teenagers have the awareness and the motivation, it is important to involve experts and use PEGASO system to support the behaviour change process and reinforce existing virtuous behaviours. PEGASO takes a holistic approach involving the teenager's environment and specifically the families, by means of an education process empowered by training that will be provided on location (schools) and on line. 


\section{System Architecture}

Figure 4 represents the architectural scheme of the PEGASO system. The system is designed to be modular, starting from the main device and key functionalities of the application, adding on additional peripherals, more complex services, feedback functions and information.

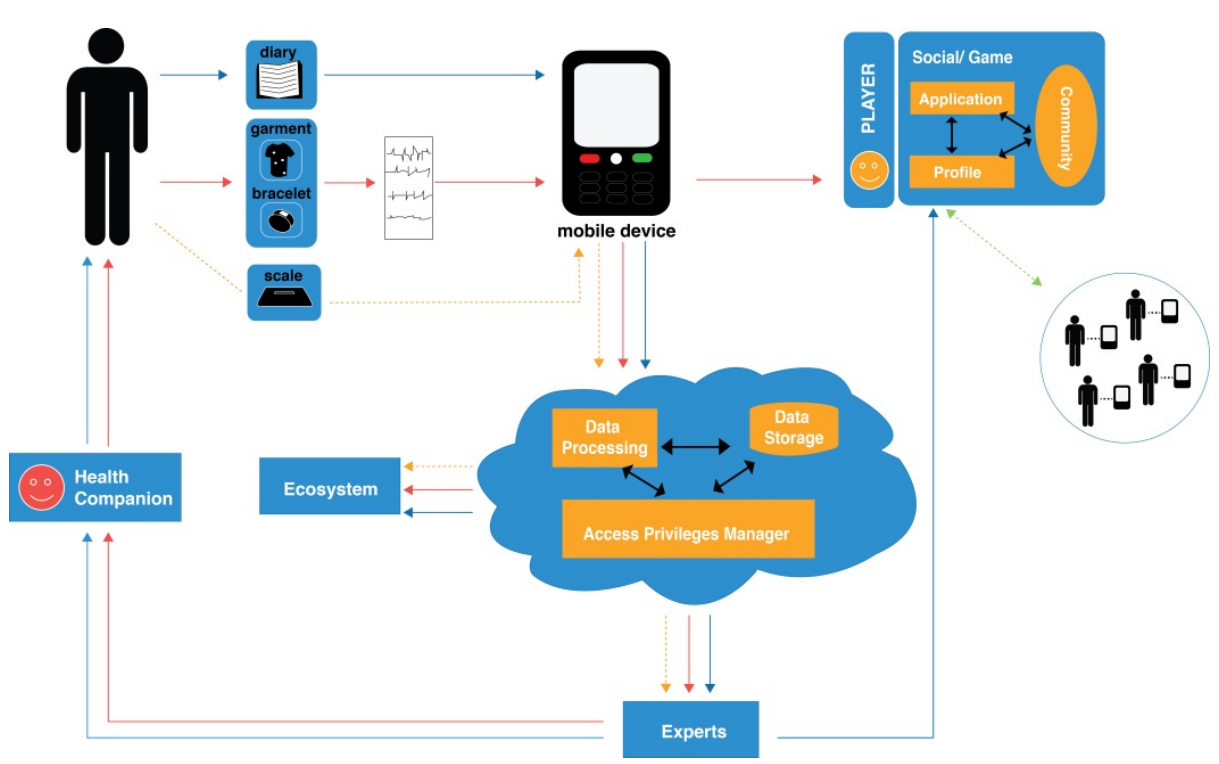

Fig. 4. The PEGASO system Architecture

PEGASO is based on a mobile platform, where the smartphone is the first and key sensor system. The mobile device also acts as communication gateway towards the other sensors. Basic services, such as those related to location (positioning based on GPS and additional algorithms to infer position in the absence of the GPS signal) and basic motion sensors to detect physical activity, are provided through sensors embedded within the smartphone.

Additional sensors that will involve additional hardware, can be added to the system: a bracelet that monitors physical activity; a scale (or balance board) that provides information about weight and body composition; specific sensors to monitor fitness activities.

The behavioural and nutritional data flow (Figure 5a) represents the information that the user provides about its nutritional habits (food intake). These data must be collected through the APP's user interface (UI) directly from the users (e.g. pictures, questionnaires, diaries, vocal annotations etc.).

The physiological data (Figure 5b) can be acquired continuously (this means, accelerometers for the bracelet and, when used, the bio signals from the garment). 

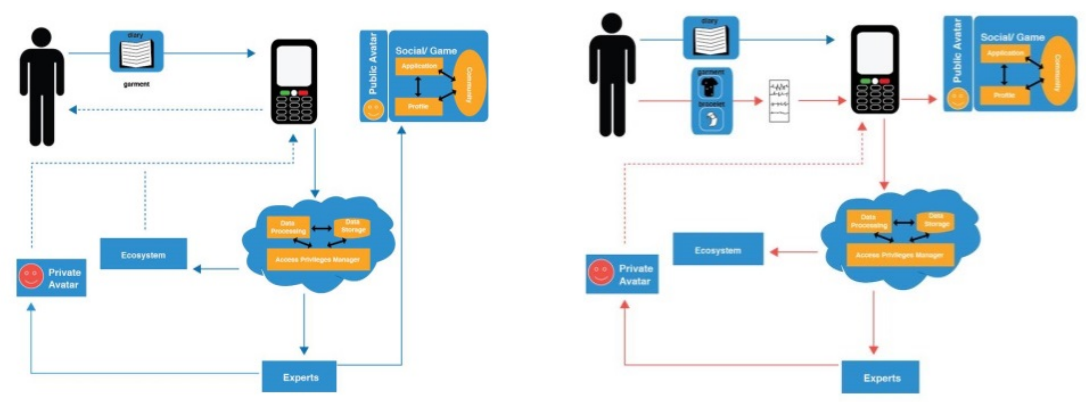

Fig. 5. a) On the left, the Behavioural dataflow of the PEGASO system; b) on the right, the physiological dataflow of the PEGASO system

Raw data coming from sensors are sent to the mobile, and processed by specific algorithms integrated inside the app. Results from processing provide two classes of processed data. The first one is sent to the Health Companion and influences the Player response inside the game. The second dataflow is sent directly to the cloud. These data should be more structured and collect more information because of their use by experts and ecosystem stakeholders. Moreover, a Multimedia Diary Platform is used for behaviour and surrounding environmental data monitoring. Diaries represent the UI to collect data concerning psychological and nutritional behaviours. Behavioural habits and information are sent to the cloud and, consequently, to ecosystems stakeholders and experts. Experts evaluate the user's nutritional habits and provide specific information directly to the Social/Game level. This information modifies the Player's characteristics and produce benefits/penalties at the community level. Experts must filter this information to avoid false information. Another data flow is sent to the Health Companion directly from experts to provide habit suggestions and evaluation of the user's lifestyle and results.

\section{System Integration}

This section briefly presents the main aspects that will be considered in the PEGASO Project with respect to interconnecting all the software applications and computing systems, so that a whole platform will be obtained.

The envisioned mobile, social and networked platform will be a collaborative one, providing cloud-based services.

From a hardware point of view, the PEGASO platform will integrate different mobile devices (smartphones, bracelets, etc.), computing systems and database systems, by using specific communication protocols. These aspects are beyond the scope of this paper but they will obviously constitute the foundation for cloud Infrastructure as a Service (IaaS).

In terms of software, PEGASO will have to offer several mobile applications and also at least one web application. While the mobile apps are essential to linking teenagers with devices and sensors, the web application will serve as a central point that 
will allow for "working together". This is the defining characteristic of a Collaborative Platform. The PEGASO web application will allow its users to network. Sensor data will be available to users from the first moment they started using PEGASO. They will be able to track their progress, to share some of their data with others, to discuss, to compare, to receive advices (from friends, from experts), to collaborate in general.

The PEGASO Project will have to manage a cloud-based system integration because the platform will be provided as a service (PaaS - Platform as a Service). This kind of system integration is required for Software as a Service (SaaS) applications.

Some of the main characteristics of cloud-based system integration are: (1) deploying in an elastic cloud infrastructure, (2) no software development (every piece of software is available and ready to be connected to another one), (3) users do not manage the platform and (4) system management and monitoring services.

From a software engineering point of view, the PEGASO system will adopt the commonly used Continuous Integration technique. It is part of the extreme programming, agile methodology, and it essentially involves: test-driven development, maintaining a code repository, automating the build process and automating the deployment process. While this technique demands well defined test cases and a significant initial setup time, the idea is to offer continuous delivery and quality assurance.

Software tools like $[21,22,23]$ will help towards PEGASO system integration. The orchestration will have to be managed properly as PEGASO will encompass a multitude of hardware devices (smartphones, tables, sensors, etc.), operating systems, communication protocols, web browsers, software applications and servers.

As described above, system integration is will assemble and package manufactured parts in successive phases in order to obtain more complete and more optimized systems allowing them to work together in the PEGASO scenarios.

In order to guarantee modularity and scalability, the integration framework will be made up by Open Source technologies in order to make the PEGASO ecosystem a real open platform, easy to up-grade and able to self-adapting very quickly both to technical progress and future mobile devices.

The integration framework will be structured by the following layers:

- Prototypes integration layer: this layer will integrate the prototypes such serious game, smart life companion, social network, bracelets, wearable sensors;

- Integration software layer: this is the main core framework software. It will provide libraries and programs to be employed by the devices and mobile/web prototypes in order to interface externally and with the API of the common communication interface layer;

- Common communication interface layer: it will define a common language in order to make the prototypes able to communicate, through Open Data protocol, with the different software modules of PEGASO ecosystem. 


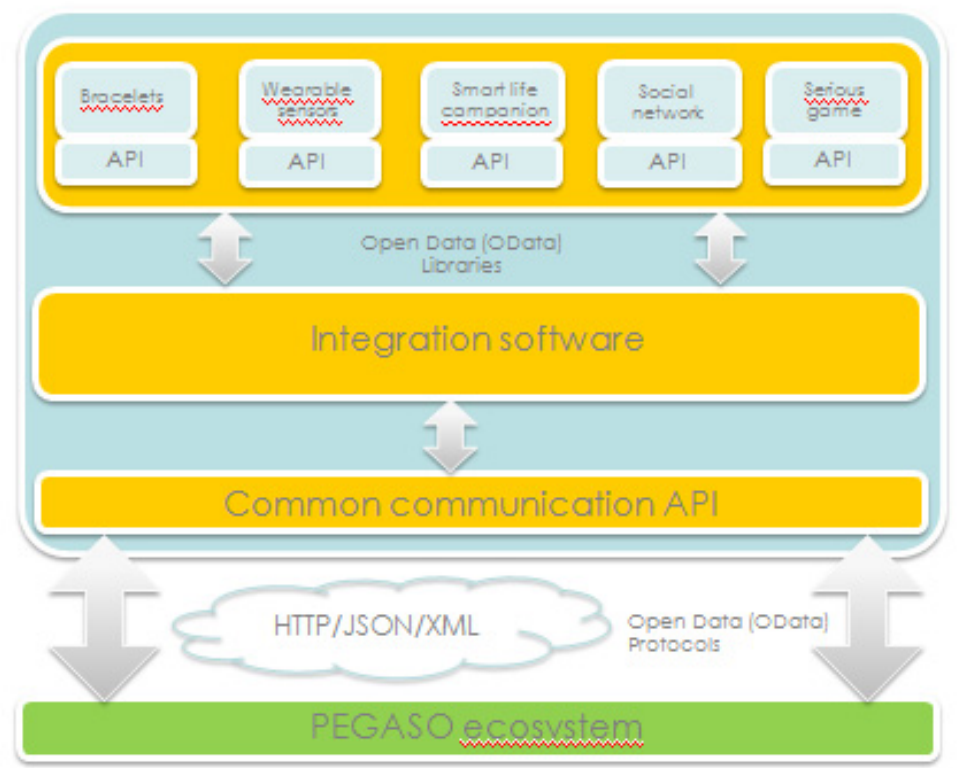

Fig. 6. System Integration

A complete definition of the common communication interface (API), having into account existing standards and interoperability with other components, will be performed and developed.

Interfaces' modules will be:

- Open Data Libraries: will provide information exchange among devices serious game, smart life companion, social network, bracelets, wearable sensors prototypes;

- $\quad$ Open Data Protocols: HTTP / JSON /XML;

- Web services: provide devices to interface with PEGASO ecosystem.

Communication interfaces of developed prototype will be connected with the whole PEGASO ecosystem through actual existing standards communication (3G or Wi-Fi network) providing both synchronization and data interchange. The architecture that will be developed will be modular in order to provide, during the prototype development, to fit efficiently to PEGASO system needs.

\section{$5 \quad$ Conclusion and Discussion}

In this paper, the PEGASO system Architecture has been presented. The PEGASO project is aimed at the development of a mobile-based platform whose aim is to generate awareness in teen agers about healthy behaviour in nutrition and lifestyles, and to create a social-based platform to motivate and engage the users to learn healthy 
habits. The progresses proposed by the PEGASO systems are related to the integration of different approach to education, such as the use of mobile media and technologies in healthcare, serious gaming approaches and location based services. The system architecture will be defined during the first year of the project and a UCD approach will be conducted to define the Users' requirements. The system integration definition will guarantee the reliability of the ICT platform and the technological frames.

\section{References}

1. WHO, http://www. euro. who.int/en/health-topics/noncommunicable diseases/obesity/obesity

2. Reilly, J.J., Methven, E., McDowell, Z.C., et al.: Healthconsequences of obesity. Archives of Disease in Childhood 88(9), 748-752 (2003)

3. Freedman, D.S., Khan, L.K., Dietz, W.H., Srinivasan, S.R., Berenson, G.S.: Relationship of childhood obesity to coronary heart disease risk factors in adulthood: The Bogalusa Heart Study. Pediatrics 108(3), 712-718 (2001)

4. Weiss, R., Caprio, S.: The metabolic consequences of childhood obesity. Best. Pract. Res. Clin. Endocrinol. Metab 19(3), 405-419 (2005)

5. Swartz, M., Puhl, R.: Childhood obesity: A societal problem to solve. Obesity Reviews 4(1), 57-71 (2003)

6. Baranowski, T., Frankel, L.: Let's Get Technical! Gaming and Technology for Weight Control and HealthPromotion in Children, Childhood Obesity 34-37 (February 2012)

7. Wright, J.D., Wang, C.Y., Kennedy-Stephenson, J., Ervin, R.B.: Dietary intake of ten key nutrients for public health, United States: 1999-2000. Advance Data no. 334, 1-4 (2003)

8. Patrick, K., Calfas, K.J., Norman, G.J., et al.: Randomized controlled trial of a primary care and home-based interventionfor physical activity and nutrition behaviors: PACE+ for adolescents. Archives of Pediatrics \& Adolescent Medicine 160(2), 128-136 (2006)

9. World Health Organization. Population-based prevention strategies for childhood obesity: Report of a WHO forumand technical meeting, Geneva, 15-17 (December 2009)

10. Timmons, B.W., Leblanc, A.G., Carson, V., et al.: Systematic Review of physical activity and health in the early years (Aged 0 -4). Appl. Physiol. Nutr. Metab. 37, 773-792 (2012)

11. Chaput, J.-P., Lambert, M., Mathieu, M.-E., Tremblay, M.S., O’Loughlin, J., Tremblay, A.: Physical activity vs. sedentary time: Indepent associations with adiposity in children. Pediatric Obesity 7, 251-258 (2012)

12. Baranowski, T., Davis, M., Resnicow, K., Baranowski, J., Doyle, C., Lin, L.S.,... Wang, D.T.: Gimme 5 Fruit, Juice, and Vegetables for Fun and Health: Outcome Evaluation. Health education \& behavior 27(1), 96-111 (2000)

13. Bonato, P.: Wearable Sensors and Systems. IEEE Engineering in Medicine and Biology Magazine 29 29(3), 25-36 (2010)

14. http://www.fitbit.com

15. http://kickinkitchen.tv

16. http://appsforheal thykids. challengepost.com

17. http://www. escapefromdiab.com

18. Sanders, E.B.: From User-Centered To Participatory Design Approaches. Design, 1-7. Taylor \&Francis (2002) 
19. Clark, N.M., Patridge, M.R.: Strengthening Asthma Education to Enhance Disease Control. Chest 121(5), 1661-1669 (2002)

20. Fogg, B.J.: A Behavior Model for Persuasive Design. In: Proceedings of the 4th International Conference onPersuasive Technology Persuasive (2009) ISBN:9781605583761

21. Hudson, http://hudson-ci.org/

22. Jenkins, http://jenkins-ci.org/

23. Rational Team Concert, https://jazz.net/products/rational-teamconcert/ 